\title{
The Effect of Alpha Lipoic Acid (ALA) on $S$. cerevisiae Cell Proliferation
}

\author{
Henna Purewal* \\ Biotechnology High School, United States \\ *Corresponding author: henna.purewal@gmail.com
}

\begin{abstract}
This experiment investigates what effect ALA may have on the growth of $S$. cerevisiae, based on extensive studies that have been conducted previously on the therapeutic capabilities of ALA ( $\alpha$-lipoic acid) in various cancers because of its potent antioxidant and apoptotic effects. It manages this because it serves a significant role in enzymatic processes crucial to energy production in cells. In energy metabolism, ALA is responsible for generating acetyl-CoA from pyruvate, and it thus plays a large role in the PDC (pyruvate dehydrogenase complex), an important link connecting glucose metabolism with the citric acid cycle [1]. It helps to shuttle carbon into aerobic respiration, specifically for converting pyruvate into acetyl-CoA and the formation of NADH and carbon dioxide. The process is regulated by enzymes called PDKs, which serve to catalyze suppression of the PDC when the cellular environment becomes nutrient-limited, or glucose scarce. PDKs are speculated to be able to inactivate the PDC via phosphorylation, thus preserving pyruvate for a different metabolic pathway. It is known that overexpression of PDKs blocks oxidative decarboxylation of pyruvate, so targeting the inhibition of PDKs could upregulate PDC activity [2]. Studies have revealed that PDC inhibition is associated with the mechanisms of many metabolic disorders, even cancer (Stacpoole, 2017). In S.cerevisiae cells, which are model organisms for cancer studies, energy is generated typically anaerobically through alcohol fermentation, until this is impacted and it switches to metabolism controlled by the citric acid cycle. This slows down cell proliferation, so it is predicted that as ALA concentrations are increased, cell proliferation will decrease, as indicated by an average cell count of each culture. After experimentation, results revealed that each ALA concentration, $0.125 \%\left(2.37 \times 10^{-20}\right), 0.25 \%\left(1.49 \times 10^{-16}\right)$, $0.5 \%\left(6.41 \times 10^{-16}\right)$, and $1 \%\left(9.36 \times 10^{-18}\right)$, significantly reduced the cell count of their respective cell culture, as compared to the control of $0 \%$ ALA. Also, alcohol test strips revealed that with higher concentrations, the cells switched to aerobic respiration as no alcohol was being produced, so fermentation was stopped. This supports the hypothesis that increasing concentrations of ALA will lead to a decrease in cell proliferation, and may indicate that ALA upregulates PDC activity via inhibition of PDKs, which makes it a potential candidate in adjuvant therapies for cancer treatment.
\end{abstract}

Keywords: PDKs, PDC, aerobic respiration, citric acid cycle, energy metabolism, alpha-lipoic acid, ALA, S. cerevisiae, cancer

Cite This Article: Henna Purewal, "The Effect of Alpha Lipoic Acid (ALA) on S. cerevisiae Cell Proliferation." American Journal of Medical and Biological Research, vol. 6, no. 2 (2018): 21-26. doi: 10.12691/ajmbr-6-2-1.

\section{Introduction}

In discussing cancer and its adaptations from a normal cell, it is important to note that perspectives are changing about its behavior as research has been on the rise in treating cancer as a metabolic disorder. In order to ensure their survival and uncontrolled growth, cancer cells reorganize their metabolic pathways in limiting environments, characterized by factors such as nutrient restriction. This mechanism for energy metabolism is explained by the Warburg Theory, which suggests that cancer cells convert glucose to lactate even in the presence of oxygen, known as aerobic glycolysis [1]. This has repeatedly been supported by many different cancer cell types, and it reveals that the process is largely inefficient compared to the oxidative phosphorylation of glucose to carbon dioxide and water. Complete oxidation of glucose results in 32 ATP molecules, while aerobic glycolysis results in only 2 ATP molecules. Cancer cells, however, have an upregulation of glucose metabolism, so in order to metabolize large amounts of glucose for rapid cell proliferation, the metabolic process is quick and employs specific adaptations to achieve this. For example, PDK (pyruvate dehydrogenase kinase) is upregulated in many cancer cell types, so the inhibition of PDC (pyruvate dehydrogenase complex) via shifting the pathway toward complete cose oxidation is speculated to eradicate tumors [3]. In studying cancer as a metabolic disorder, targeting the aerobic glycolysis pathway holds potential value as a therapeutic stratagem.

In normal cells, the PDC shuttles carbon into the ETC to produce greater amounts of ATP, but the process 
produces ROS (reactive oxygen species) which can result in oxidative damage. Normal cells, however, prepare protection from this damage through utilizing anti-oxidants. Cancer cells, meanwhile, do not employ the means for protecting themselves from oxidative damage which makes them susceptible to oxidative molecules, such as ALA. Therefore, cancer cells inhibit the PDC to prevent carbon shuttling into the ETC, which is a key feature that recent research is beginning to target, as it would mean a damaged mitochondria which would then lead to apoptosis as cancer cells have no means in acquiring energy [3]. Alpha lipoic acid, a key molecule in the PDC, can perform this function as it inhibits PDK, thus upregulating the PDC. This action would force cancer cells to shuttle carbon into the ETC, and as stated before, it would result in mitochondrial damage and issue apoptosis shortly after.

Essentially, the aim of this investigation is inspired by the knowledge that the PDC converts pyruvate to acetyl-CoA via decarboxylation to prevent lactate formation, which is downregulated in most cancers while PDKs, regulators of this process, are upregulated in order to suppress the PDC. Knowing this, it is curious to find whether ALA (alpha lipoic acid) can activate the PDC in order to reverse the effects of aerobic glycolysis and promote apoptosis.

With association to metabolic disorders, such as diabetes, ALA has served as a known antidiabetic by counteracting the "massive oxidative and inflammatory changes the disease produces". It is suggested that because of ALA's powerful role as an antidiabetic, its effects are evidenced to extend to obesity-related cancers including breast cancer, thyroid cancer, colorectal cancer, pancreatic cancer and liver cancer. On the molecular level, ALA serves as a cofactor for many enzymatic processes involved in the citric acid cycle and the PDC, where it aids in the decarboxylation of pyruvate to acetyl-CoA, a necessary step between glycolysis and the citric acid cycle [4]. For this reason, ALA is tested for the effects of its different concentrations on a well-studied model organism for cancer studies, $S$. cerevisiae.

S. cerevisiae (Saccharomyces cerevisiae) is a well-researched model organism that is utilized as a simpler representation of the systems and biological pathways found in human cells. For this, yeast is known to be a close metabolic homologue to human cells as they share high genetic similarity in basic metabolic functions [5]. S. cerevisiae is an accurate model in basic cellular structure and function as both human cells and yeast are eukaryotic organisms. For example, in cellular respiration [6], the reactants and products involved in the process, such as the decarboxylation of pyruvate into acetyl-CoA and the ETC in $S$. cerevisiae mitochondria are consistent with the processes found in human cells, as are some of the enzymes involved, such as PDKs. Knowing this, $S$. cerevisiae is an economical choice as a representative of this investigation into cancer cell metabolism, and for its rapid period of growth, making the experiment much more feasible. Also, as an indicator of whether the PDC is active or not, S. cerevisiae cells revert to alcohol fermentation when their primary aerobic pathway is blocked. As such, if it undergoes alcohol fermentation, then it would suggest that the PDC is not activated, whereas if it is not producing alcohol, then the PDC is active, This is the reasoning behind the dependent variable of the experiment as cell proliferation decreases, then it may suggest that the PDC is active. A simple alcohol test-strip will be used to validate this. If increasing concentrations of ALA are applied to cultures of $S$. cerevisiae cells, thus activating the PDC and reverting back to an aerobic pathway versus alcohol fermentation, then the cells will proliferate at a slower rate than cells cultured in smaller concentrations of ALA. In order to measure the difference in proliferation, a hemocytometer will be used to provide a representative estimate of the cell culture, via using a cell counter to manually count the number of cells on two quadrants of the grid on the hemacytometer. These cell counts will then be averaged for each concentration to provide an estimate of the cell count in $10 \mu \mathrm{L}$ of a $5 \mathrm{~mL}$ culture.

\section{Materials}

The materials used for this experiment include: magnetic stirrer, alpha lipoic acid capsules (ALA), conical tubes, autoclave tape, screw-cap tubes, sterile inoculating loops, YPD broth powder, 125-mL media bottles autoclave, lighter, distilled water, autoclave, scale, micropipets and tips, serological pipets, hemocytometer, cell counter, 70\% ethanol, Kimwipes, Trypan Blue, wild-T S. cerevisiae, $37^{\circ} \mathrm{C}$ incubator, compound microscope, and alcohol test strips.

\section{Methods}

\subsection{Making YPD Broth}

To prepare $155 \mathrm{mLs}$ of YPD broth, distilled water and YPD broth powder were used, based upon the listed ratio provided by the YPD broth product. The provided ratio was 50 grams per $1,000 \mathrm{~mL}$ of water, so to make $155 \mathrm{~mL}$ of broth, it was calculated that 7.75 grams of broth powder should be suspended in $155 \mathrm{mLs}$ of distilled water in two $125 \mathrm{~mL}$ media bottles. Contents were mixed using a magnetic stirrer, and after about 3 minutes when the broth powder appeared dissolved, and the stirrer was removed, the bottles were appropriately labeled with autoclave tape applied as they were autoclaved for sterilization.

\subsection{Preparing Cell Culture- Inoculating S. cerevisiae}

Using two conical tubes with air-tight seals and a serological pipet, $5 \mathrm{mLs}$ of sterilized YPD broth was transferred to each of the two conical tubes. For each tube, an inoculating loop was used to transfer an estimated $1 \mathrm{~mm}$ x $1 \mathrm{~mm}$ colony from a stock tube of $S$. cerevisiae and into each tube. The tubes were then sealed with their lids and incubated at $37^{\circ} \mathrm{C}$ for 24 hours, maximum for proper harvesting.

\subsection{Preparing ALA Concentrations}

To prepare $0.125 \%, 0.25 \%, 0.5 \%$, and $1 \%$ ALA concentrations, the ratio provided by the ALA capsule 
product was used to calculate the amount of ALA for 30 mLs of each concentration. For $0.125 \%$ ALA, 0.038 grams of ALA was mixed with 1.5 grams of YPD broth powder. For $0.25 \%$ ALA, 0.075 grams of ALA was mixed with 1.5 grams of YPD broth powder. For $0.5 \%$ ALA, 0.15 grams of ALA was mixed with 1.5 grams of YPD broth powder. For 1\% ALA, 0.3 grams of ALA was mixed with 1.5 grams of YPD broth powder. Mixtures were made in $100 \mathrm{~mL}$ media bottles and stirred using a magnetic stirrer and plate, until after about 5 minutes when the solutions appeared evenly distributed, the bottles were labeled accordingly, autoclave tape was applied, and the bottles were autoclaved for sterilization.

\subsection{Applying ALA Treatment to $S$. cerevisiae Culture}

25 screw-cap, gamma sterilized tubes were prepared by transferring $5 \mathrm{mLs}$ of each ALA concentration into their 5 respective tubes, with $1 \mathrm{~mL}$ of $S$. cerevisiae culture in every tube. Tubes were labeled prior to transfer, with the ALA concentration and the trial number. There were 25 tubes total, with 5 replicates for each of the five concentrations. The negative control in the experiment was $0 \%$ ALA, while other concentrations were $0.125 \%$, $0.25 \%, 0.5 \%$, and $1 \%$ ALA. After applying the ALA treatment to the respective tubes, all 25 tubes were placed in an incubator set to $37^{\circ} \mathrm{C}$ for 24 hours. Following incubation, one representative sample from each concentration was tested for fermentation using alcohol test strips to detect whether alcohol was present in the culture.

\subsection{Experimental Variables}

The independent variable investigated in this experiment is ALA concentrations, $0.125 \%, 0.25 \%, 0.5 \%$, and $1 \%$, while the dependent variable is the cell count of $S$. cerevisiae in two quadrants of the hemocytometer grid. All other variables were controlled for the duration of the entire experiment so as to ensure accuracy in results. The temperature for stock cell culture incubation and treated culture incubation was consistently $37^{\circ} \mathrm{C}$ for 24 hours maximum. The same yeast strain was used for each trial, wild-type S. cerevisiae, and the broth medium (YPD) used for the stock cell culture was also used for the treated cell culture tubes. The same amount of YPD broth medium was also added into each stock concentration, 1.49 grams, and the volume for each concentration was kept consistent at $30 \mathrm{mLs}$. The volume of $\mathrm{S}$. cerevisiae culture added to each treatment tube was also kept constant at $1 \mathrm{~mL}$.

\subsection{Microscopy and Hemocytometer [7]}

In order to examine $S$. cerevisiae growth using a compound microscope, 25 microcentrifuge tubes were labeled accordingly with a respective trial number and corresponding ALA concentration following transfer of the treated cultures. To each tube, $50 \mu \mathrm{L}$ of a respective cell culture was added with $10 \mu \mathrm{L}$ of Trypan Blue solution to stain dead cells so that differentiation from living cells was possible. Every tube was then inverted few times to mix the sample. A hemocytometer was then prepared using 70\% ethanol and a Kimwipe to sterilize the grid surface before transferring a sample culture. This was performed 25 times, once before analyzing every sample. When transferring a sample culture, the glass slide was first cleaned and then gently placed over the hemocytometer grid, and $10 \mu \mathrm{L}$ of a sample was transferred from its respective microcentrifuge tube and into the chamber between the glass slide and the hemocytometer plate. Once focusing the microscope onto the grid, which was divided into 4 quadrants, cells were counted using a cell counter in the top two quadrants. This was kept consistent for every trial. The average cell count was then found, and a representative estimate for each culture.

\subsection{Statistical Analysis}

To effectively exhibit the data found, the cell count for each ALA concentration was averaged for all five replicates in each of the four treatment groups and control, thus an average sample cell count was found. Standard deviations were then calculated along with the average cell count, Average cell counts were displayed visually using a bar graph with error bars representing one standard deviation, and an asterisk atop every ALA concentration bar for those which revealed a p-value less than 0.05. This indicated that a specific ALA treatment was statistically significant in its difference as compared to the control. The ALA treatment concentration was plotted on the $\mathrm{x}$-axis according to the concentrations of $0 \%, 0.125 \%, 0.25 \%, 0.5 \%$, and $1 \%$, while the average cell count was plotted on the y-axis. T-tests were performed using Microsoft Excel to compare every ALA treatment concentration to the control in order to determine whether there was significant variation from every treatment group.

\section{Results}

Raw data was processed by calculating the average cell count for each ALA concentration, representing the cell count of each sample culture as in two quadrants of the hemocytometer grid. As shown in Table 1, the average cell counts revealed a large decrease as ALA concentrations increase. Standard deviations reveal variability in the data, which is also shown to decrease with increasing concentrations because decreasing cell counts narrowed the cell count range.

Table 1. The Effect of ALA Treatment on the Cell Count of S. cerevisiae Using a Hemocytometer

\begin{tabular}{|c|c|}
\hline ALA Concentration (\%) & Average Cell Count \pm Standard Deviation \\
\hline 0 & $774 \pm 234.8$ \\
\hline 0.125 & $90 \pm 33.5$ \\
\hline 0.25 & $48 \pm 7.6$ \\
\hline 0.5 & $31 \pm 11.8$ \\
\hline 1.0 & $37 \pm 14.6$ \\
\hline
\end{tabular}

For the respective ALA concentration $(0 \%, 0.125 \%$, $0.25 \%, 0.5 \%, 1.0 \%)$, the corresponding average raw cell count and standard deviation is listed. The average raw cell count is the average cell count of two quadrants using a hemocytometer in all five trials per ALA concentration. In total, 25 trials were performed with 5 replicates per concentration, thus 5 trials per concentration. 


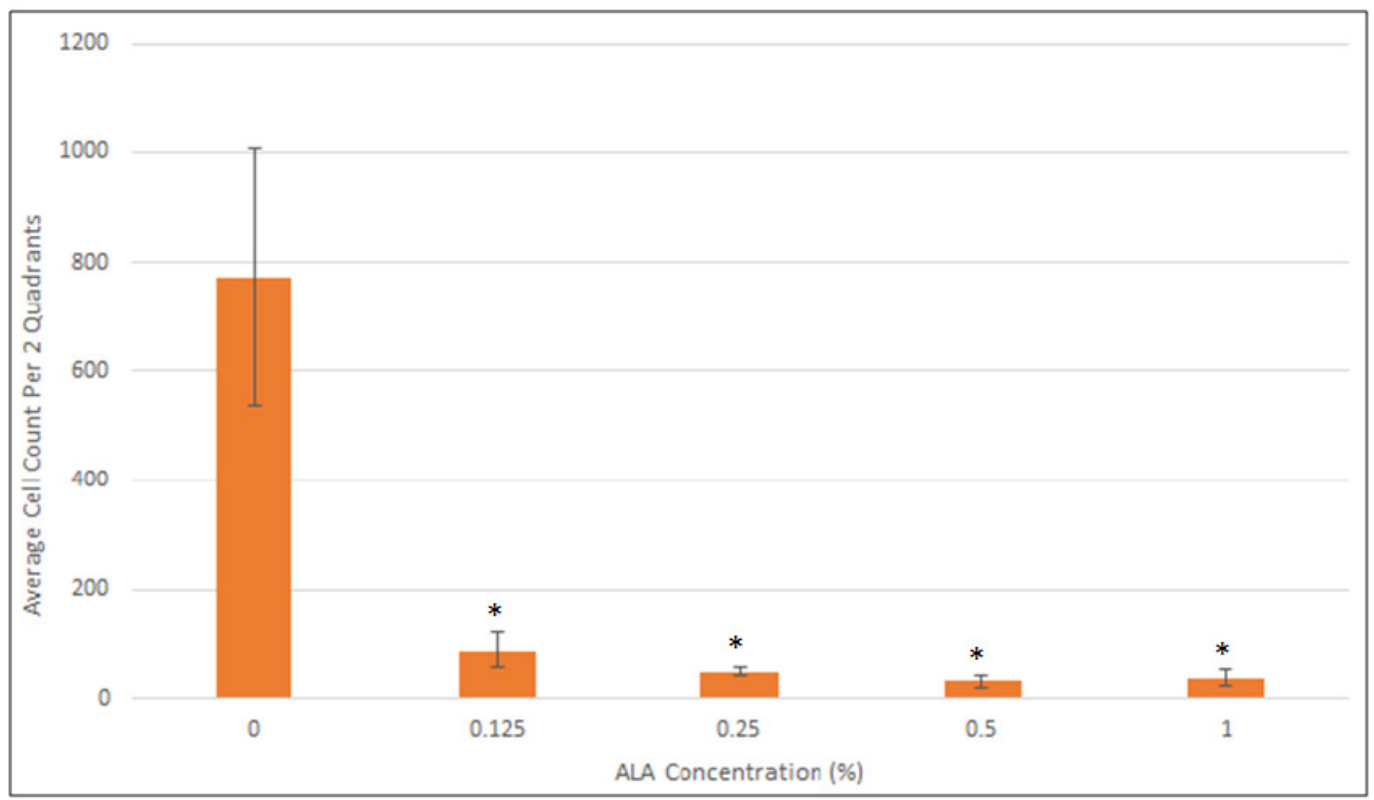

Figure 1. The Effect of ALA Treatment on Average Cell Count Per 2 Quadrants of $S$. cerevisiae Cells

T-tests were then calculated between the control of $0 \%$ ALA and each treatment concentration, $0 \%, 0.125 \%$, $0.25 \%, 0.5 \%, 1.0 \%$. By performing a t-test, it would reveal whether there was a significant difference between the average raw cell counts of the control and each treatment concentration, because although there is variation, it may not be significant. The t-tests were performed using Microsoft Excel, and were one-tailed, type 3 distributions because a decrease in cell count was expected between the control and the treatment concentration. The p-values and significance are indicated in Figure 1, which exhibits the average raw cell count for each ALA concentration and the standard deviation from the average, shown as error bars with one error bar being equivalent to one standard deviation.

Processed data from Table 1 is displayed graphically in this figure, with the ALA concentrations on the $\mathrm{x}$-axis and the average raw cell count is displayed on the y-axis. Each bar represents a respective concentration, with the error bars representing the standard deviation as shown in Table 1. An asterisk over each bar is representative of the a p-value less than 0.05 for the respective ALA concentration, as compared to the control, which was determined through performing t-tests between each concentration and the control of $0 \%$ ALA.

Alcohol tests were then performed for a single sample from each treatment concentration group, and they revealed that with lower concentrations, specifically $0.125 \%$ and the control, alcohol fermentation took place, but as concentrations increased, $0.25 \%, 0.5 \%$, and $1 \%$, no alcohol was detected in the sample culture, revealing fermentation was inactive and the aerobic pathway involving PDC and the citric acid cycle were active. The image below, Image 1, exhibits this finding.

Using alcohol test strips, a sample culture was selected from each ALA concentration and tested for alcohol presence. For concentrations $0.25 \%, 0.5 \%$, and $1 \%$, no alcohol was detected, while for the control and $0.125 \%$ ALA, $0.30 \%$ and $0.08 \%$ alcohol was detected respectively. This reveals that fermentation was indeed active, even at a lower concentration, but it was inactive as ALA concentrations increased in the cell culture environment, which must have forced the cells into using an aerobic pathway involving the PDC and the citric acid cycle as no alcohol was detected with higher concentrations.

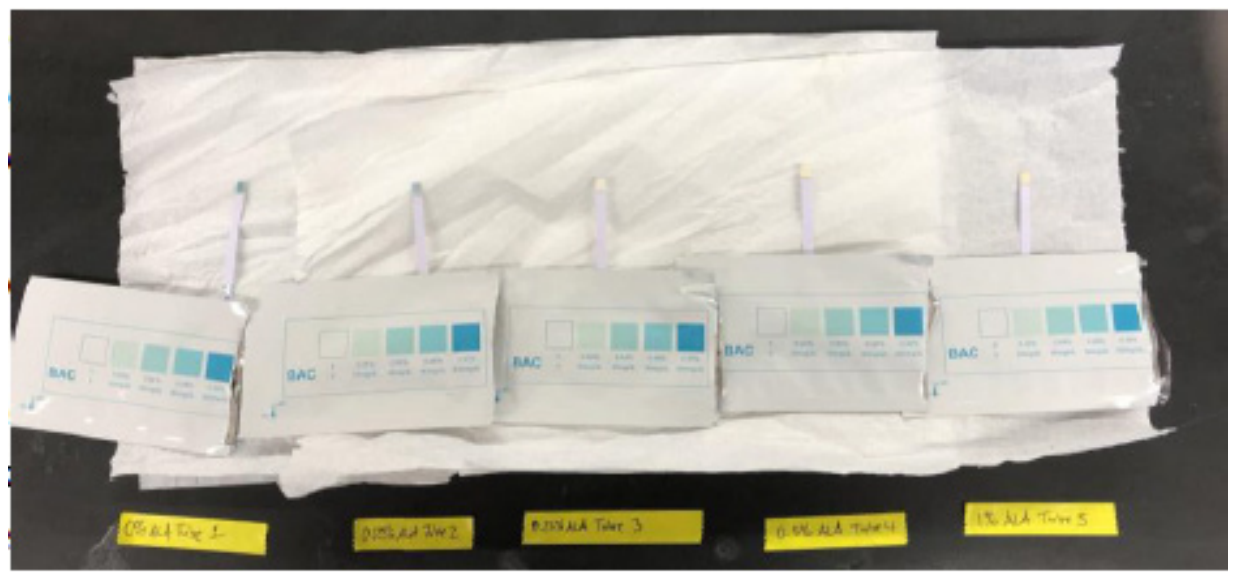

Image 1. Alcohol Presence in S. cerevisiae Sample Cultures 


\section{Conclusion}

The hypothesis that as ALA concentrations increase, $S$. cerevisiae cell proliferation, measured as cell count, will decrease is supported by the results found. Figure 1 effectively exhibits a decline in the average raw cell for every increasing ALA concentration. As Figure 1 also shows with an asterisk above each ALA concentration treatment, $0.125 \%\left(2.37 \times 10^{-20}\right), 0.25 \%\left(1.49 \times 10^{-16}\right), 0.5 \%$ $\left(6.41 \times 10^{-16}\right)$, and $1 \%\left(9.36 \times 10^{-18}\right)$, ALA significantly reduced the $S$. cerevisiae cell count as it is compared to the control. These results may be due to the effect of ALA in upregulating PDC via inhibition of PDKs in the cells. As ALA increases in the cellular environment, fermentation becomes inactivated as its effects on PDKs would have reverted the cells back to aerobic respiration, using complete oxidation of glucose and thus activating the citric acid cycle and the PDC. This is supported by the results shown in Image 1 where alcohol is shown to be present at $0 \%$ ALA (control), revealing active alcohol fermentation, and at $0.125 \%$ ALA, the lowest concentration tested. Beyond this, all concentrations tested revealed no alcohol presence, indicative that fermentation was inhibited. A clear conclusion can be made, therefore, that ALA inhibited fermentation and induced aerobic respiration via the PDC and citric acid cycle in $S$. cerevisiae cells.

With ALA inhibiting PDKs, and thus activating the PDC, this would have resulted in mitochondrial damage in the S. cerevisiae cells. As a result of mitochondrial damage due to ROS generation, cell proliferation would decrease as apoptosis would be triggered as cells would be without the means for ATP production [8]. However, cell counts remained quite similar to each other on average, for example, for $0.5 \%$ and $1 \%$ ALA, the average raw cell count was 31 and 37 cells respectively, which may be because ALA plays its role in the enzymatic processes involved with the PDC, so there must be a plateau in declining cell count with increasing concentrations of ALA as there is a limited amount of substrates, pyruvate, for the metabolic pathway.

Knowing the effects of higher concentrations of ALA on the growth of S. cerevisiae cells, in that they may decrease cell proliferation via inhibition of PDKs, as shown by inhibition of fermentation, further application can be made toward cancer research. As previous research studies have shown, PDKs are upregulated in many cancer cell types, because it allows cells to protect itself under nutrient-limited environments and metabolize other substances such as lipids in order to ensure rapid cell proliferation [1]. This experiment shows that ALA concentrations as low as $0.125 \%$ can control proliferation and significantly limit it, as compared to there not being any concentration applied. When lactate or alcohol is not being produced, this is indicative of active PDC and downregulated PDKs, so it may be states that ALA concentrations from $0.125 \%$ and above have the capacity to control cell proliferation, and may be applied as an adjuvant treatment of certain cancers via inhibition of PDKs.

If this were the case, tumor sizes may be reduced via adjuvant ALA treatments for cancer patients, in an effort to induce apoptosis in the cancer cells. If this treatment were employed, then not only will PDKs be inhibited, but the PDC and aerobic pathway would be upregulated and lead to mitochondrial damage. Cell proliferation would slow, and tumors may begin to shrink, but as for healthy cells in the body, unaffected by cancer, they may not be affected as they employ a range of protective mechanisms against mitochondrial damage which may be induced, although a slight chance of mutations may still exist.

Throughout the experiment, there were many potential sources of error that could have affected the integrity of the results found. A source of error with the greatest chance of occurring was contamination of the samples. Contamination, fortunately, did not occur in this experiment, but the chances would have been quite high, as a fume hood could not have been used to ensure sterile conditions, as yeast is a contaminant to the fume hood itself. To avoid contamination, disposable sterile inoculating loops were used for inoculation of the culture. Also, airtight conical tubes were used to prevent contamination from microbes in the air, but it would not have affected the results as the culture was incubated for a short period of time of 24 hours and there would have been sufficient amount of oxygen for the culture to grow. Another improvement that may be made to the experiment is the use of a spectrophotometer to give more substantial results in measuring cell proliferation, as it would do so by finding the density of the cell culture sample, and this is more accurate than simply counting cells using a hemocytometer, which lends more error.

During this experimentation, it was noted that $S$. cerevisiae cells are quite accurate models of human cellular function, and they were very easy to use as there is a quick period of growth, and are quite low-maintenance. The technique of using a hemocytometer is also very useful if performing similar research that may require an estimated cell count, although it can become taxing.

In considering a continuation of this experiment, ALA concentration treatments could be applied to different cancer cell types, as opposed to testing them on $S$. cerevisiae cells. Similar techniques would be used, but perhaps different maintenance would be required in culturing the cells, and not only may hemocytometer be used, but a spectrophotometer may also be used to provide more substantial results in measuring cell proliferation. This further experimentation may further validate specific cancers as metabolic disorders.

\section{Acknowledgements}

I would like to acknowledge Dr. Julie Nowicki for her guidance and mentorship throughout this experiment.

\section{References}

[1] Zhang, S., Hulver, M. W., McMillan, R. P., Cline, M. A., \& Gilbert, E. R. (2014). The pivotal role of pyruvate dehydrogenase kinases in metabolic flexibility. Nutrition \& Metabolism, 11, 10.

[2] Zhang, W., Zhang, S.-L., Hu, X., \& Tam, K. Y. (2015). Targeting Tumor Metabolism for Cancer Treatment: Is Pyruvate Dehydrogenase Kinases (PDKs) a Viable Anticancer Target? International Journal of Biological Sciences, 11(12), 1390-1400. 
[3] Paul M. Bingham and Zuzana Zachar (2012). The Pyruvate Dehydrogenase Complex in Cancer: Implications for the Transformed State and Cancer Chemotherapy, Dehydrogenases, Prof. Rosa Angela Canuto (Ed.), InTech, Available from: https://www.intechopen.com/books/dehydrogenases/the-pyruvatedehydrogenase-complex-in-cancer-implications-for-thetransformed-state-and-cancer-chemo.

[4] Feuerecker, B., Pirsig, S., Seidl, C., Aichler, M., Feuchtinger, A., Bruchelt, G., \& Senekowitsch-Schmidtke, R. (2012). Lipoic acid inhibits cell proliferation of tumor cells in vitro and in vivo. Cancer Biology \& Therapy, 13(14), 1425-1435.

[5] Karathia H, Vilaprinyo E, Sorribas A, Alves R (2011) Saccharomyces cerevisiae as a Model Organism: A Comparative Study. PLoS ONE 6(2): e16015.
[6] James A.Gordon,Cook Ritchard M.,West Shauna M. and Lindsay J.Gordon(1995), The pyruvate dehydrogenase complex of Saccharomyces cerevisiae is regulated by phosphorylation, FEBS Letters, 373.

[7] Massachusetts Institute of Technology. (2002). How To Use A Hemocytometer. Retrieved from

https://biology.mit.edu/sites/default/files/hemacytometer\%20activi ty\%20revised\%207-23-13.pdf.

[8] Korotchkina LG, L. (2004). R-Lipoic Acid Inhibits Mammalian Pyruvate Dehydrogenase Kinase. Taylor and Francis Online. Retrieved 16 January 2018, from

http://www.tandfonline.com/doi/abs/10.1080/10715760400004168.

\section{Appendix}

Raw Data Table A1. The Effect of ALA Concentrations on the Cell Count Per Two Quadrants

\begin{tabular}{|c|c|c|c|c|c|}
\hline ALA Concentration $\%$ & Trial & Quadrant 1 & Quadrant 2 & Average & SD \\
\hline \multirow[t]{5}{*}{0} & 1 & 754 & 748 & 774 & 234.8 \\
\hline & 2 & 808 & 787 & & \\
\hline & 3 & 755 & 739 & & \\
\hline & 4 & 757 & 798 & & \\
\hline & 5 & 792 & 805 & & \\
\hline \multirow[t]{5}{*}{0.125} & 1 & 116 & 107 & 90 & 33.5 \\
\hline & 2 & 104 & 96 & & \\
\hline & 3 & 99 & 70 & & \\
\hline & 4 & 117 & 109 & & \\
\hline & 5 & 101 & 75 & & \\
\hline \multirow[t]{5}{*}{0.25} & 1 & 43 & 33 & 48 & 7.6 \\
\hline & 2 & 44 & 52 & & \\
\hline & 3 & 48 & 59 & & \\
\hline & 4 & 44 & 47 & & \\
\hline & 5 & 57 & 53 & & \\
\hline \multirow[t]{5}{*}{0.5} & 1 & 42 & 40 & 31 & 11.8 \\
\hline & 2 & 44 & 36 & & \\
\hline & 3 & 32 & 31 & & \\
\hline & 4 & 35 & 29 & & \\
\hline & 5 & 30 & 27 & & \\
\hline \multirow[t]{5}{*}{1} & 1 & 41 & 33 & 37 & 14.6 \\
\hline & 2 & 38 & 29 & & \\
\hline & 3 & 49 & 31 & & \\
\hline & 4 & 34 & 18 & & \\
\hline & 5 & 46 & 50 & & \\
\hline
\end{tabular}

Table A2. T-test Comparing Each ALA Concentration to the Control

\begin{tabular}{|c|c|}
\hline ALA Concentration (\%) & P-Value \\
\hline 0 & --- \\
\hline 0.125 & $2.37 \mathrm{E}-20$ \\
\hline 0.25 & $1.49 \mathrm{E}-16$ \\
\hline 0.5 & $6.41 \mathrm{E}-16$ \\
\hline 1 & $9.36 \mathrm{E}-18$ \\
\hline
\end{tabular}

Table A2- This table shows the p-values for the t-tests calculated between the control (0\% ALA) and each ALA treatment concentration. It reveals that every concentration is significantly different from the control as all $p$-values are less than 0.05 . 